\title{
Erratum: Bending Rigidity of 2D Silica [Phys. Rev. Lett. 120, 226101 (2018)]
}

\author{
C. Büchner®, S. D. Eder®, T. Nesse®, D. Kuhness®, P. Schlexer®, G. Pacchioni®, J. R. Manson®,

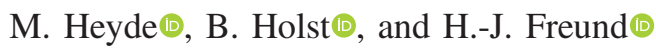

(Received 6 May 2021; published 7 July 2021)

DOI: 10.1103/PhysRevLett.127.029902

Although the results presented in this Letter reporting the measurement of the bending rigidity constant $\kappa$ of a twodimensional (2D) bilayer of $\mathrm{SiO}_{2}$ (silica) remain completely unaffected, it has been brought to our attention that in Eq. (2) we repeated an incorrect expression for the dispersion of the shear vertical (ZA) mode of a freestanding thin membrane with fixed edge (d'Alembert) boundary conditions [1]. The correct expression is

$$
\omega_{\mathrm{ZA}}^{2}(\Delta \mathbf{K})=v_{\mathrm{ZA}}^{2} \Delta \mathbf{K}^{2}+\frac{\kappa}{\rho_{2 \mathrm{D}}} \Delta \mathbf{K}^{4},
$$

where $\Delta \mathbf{K}$ is the parallel wave vector transfer, $\rho_{2 \mathrm{D}}$ is the $2 \mathrm{D}$ mass density of the membrane, $v_{\mathrm{ZA}}=\sqrt{c_{44} / \rho_{2 \mathrm{D}}}$, and $c_{44}$ is the shear force constant [2-4]. In Eq. (2) the acoustic term $v_{\mathrm{ZA}}^{2} \Delta \mathbf{K}^{2}$ was missing, which makes it to be the correct dispersion equation for a large thin freestanding membrane with free edge (Lagrange) boundary conditions. Typically, the leading term in Eq. (1) is small and the dispersion is dominated by the term in $\Delta \mathbf{K}^{4}$ except at very large wavelengths compared to the lattice spacing.

When a thin membrane is supported on a flat and ordered substrate, the ZA mode dispersion has been reported to be [5]

$$
\omega_{\mathrm{ZA}}^{2}(\Delta \mathbf{K})=\omega_{0}^{2}+\frac{\kappa}{\rho_{2 \mathrm{D}}} \Delta \mathbf{K}^{4}
$$

where $\omega_{0}$ is the gap frequency due to the coupling between the film and substrate. This is the expression used to extract, from measured $\mathrm{He}$ atom scattering spectra, the value of the flexural constant $\kappa$ for bilayer $\mathrm{SiO}_{2}$, and which has also been used similarly with monolayer graphene on several close packed metal substrates [6].

In principle, since a supported thin membrane corresponds to d'Alembert boundary conditions, a quadratic (acoustic) term similar to that of Eq. (1) should also appear in Eq. (2), but such a term is of negligible importance because it is completely dominated at small $\Delta \mathbf{K}$ by the constant term. Thus the values $\kappa$ for the bending rigidity of bilayer $\mathrm{SiO}_{2}$ as determined from our He atom scattering experiments, and all other conclusions in our Letter, remain completely unaffected. For a detailed explanation of the d'Alembert and Lagrange solutions, together with references, see Ref. [1].

We would like to thank Professor G. Benedek for bringing this problem to our attention. We would like to thank Professor Marco Bernasconi for helpful discussions. S. D. E., T. N., and B. H. acknowledge support from the Research Council of Norway, Projects No. 213453 and No. 234159. C. B. acknowledges funding from the German Research Council: Project No. 1109. Funding was also received from the European Research Council (ERC) under the European Unions Horizon 2020 research and innovation programme (Grant Agreement No. 669179).

[1] G. Benedek, J. R. Manson, and S. Miret-Artés, Phys. Chem. Chem. Phys. 23, 7575 (2021).

[2] L. D. Landau and I. M. Lifshitz, Theory of Elasticity, Third Edition, (Elsevier, Amsterdam, 1986), Sect. 25.

[3] T. Nihira and T. Iwata, Phys. Rev. B 68, 134305 (2003).

[4] D. L. Nika and A. A. Balandin, Rep. Prog. Phys. 80, 036502 (2017).

[5] B. Amorim and F. Guinea, Phys. Rev. B 88, 115418 (2013).

[6] For references, see Ref. [1].

Published by the American Physical Society under the terms of the Creative Commons Attribution 4.0 International license. Further distribution of this work must maintain attribution to the author(s) and the published articles title, journal citation, and DOI. 